\title{
Lnc-THOR silencing inhibits human glioma cell survival by activating MAGEA6-AMPK signaling
}

\author{
Jun Xue', Shan Zhong ${ }^{1}$, Bo-min Sun ${ }^{2}$, Qing-Fang Sun ${ }^{1}$, Liang-Yun $\mathrm{Hu}^{2}$ and Si-Jian Pan ${ }^{1}$
}

\begin{abstract}
Long non-coding RNA THOR (LnC-THOR) binds to IGF2BP1, essential for its function. We here show that Lnc-THOR is expressed in human glioma tissues and cells. Its expression is extremely low or even undetected in normal brain tissues, as well as in human neuronal cells and astrocytes. We show that Lnc-THOR directly binds to IGF2BP1 in established and primary human glioma cells. shRNA-mediated Lnc-THOR knockdown or CRISPR/Cas9-induced LncTHOR knockout potently inhibited cell survival and proliferation, while provoking glioma cell apoptosis. Contrarily, forced overexpression of LnC-THOR promoted glioma cell growth and migration. Importantly, Lnc-THOR shRNA or knockout activated MAGEA6-AMPK signaling in glioma cells. AMPK inactivation, by AMPKa1 shRNA, knockout, or dominant-negative mutation (T172A), attenuated Lnc-THOR shRNA-induced A172 glioma cell apoptosis. Moreover, CRISPR/Cas9-induced IGF2BP1 knockout activated MAGEA6-AMPK signaling as well, causing A172 glioma cell apoptosis. Significantly, Lnc-THOR shRNA was ineffective in IGF2BP1 KO A172 cells. In vivo, Lnc-THOR silencing or knockout potently inhibited subcutaneous A172 xenograft tumor growth in mice. MAGEA6 downregulation and AMPK activation were detected in Lnc-THOR-silenced/-KO A172 tumor tissues. Taken together, Lnc-THOR depletion inhibits human glioma cell survival possibly by activating MAGEA6-AMPK signaling.
\end{abstract}

\section{Introduction}

Glioma is among the most aggressive human malignancies, causing significant human mortalities each year ${ }^{1-3}$. In the clinical practices, gliomas are commonly diagnosed at late/advanced stages with extremely poor prognosis ${ }^{3}$. Molecularly targeted therapies are essential for better glioma prognosis ${ }^{4-6}$. Our group has been exploring novel therapeutic targets for this devastating disease ${ }^{7-10}$. Noncoding RNAs (ncRNAs), including microRNAs, long noncoding RNAs (LncRNA), and circular RNAs, are originally known as transcriptional noise. Recent studies have implied that $L n c R N A s$, and other $n c R N A s$, play pivotal roles

\footnotetext{
Correspondence: Liang-Yun Hu (luckyhu163@163.com) or

Si-Jian Pan (swordspan163@163.com)

'Department of Neurosurgery, Rui-Jin Hospital, Shanghai Jiao-Tong University School of Medicine, 200025 Shanghai, P. R. China

2Department of Stereotactic and Functional Neurosurgery, Rui-Jin Hospital, Shanghai Jiao-Tong University School of Medicine, 200025 Shanghai, P. R. China

These authors contributed equally: Jun Xue, Shan Zhong

Edited by E. Candi
}

in initiation and progression of human glioma ${ }^{11}$ and many other cancers ${ }^{12-14}$.

A recent study by Hosono et al. has reported a conserved LncRNA, THOR (“Lnc-THOR”) ${ }^{15}$. Its expression is detected in testis, and also in a number of different human cancers ${ }^{15-20}$. Lnc-THOR knockdown or knockout (KO) potently inhibited human cancer cell survival ${ }^{15}$. LncTHOR directly associates with insulin-like growth factor 2 (IGF2) mRNA-binding protein 1 (IGF2BP1), a conserved RNA-binding family protein ${ }^{15}$. Lnc-THOR association is essential for IGF2BP1's function, as well as stabilization of IGF2BP1 target mRNAs, including IGF2, Gli1 (gliomaassociated oncogene homolog 1), Myc, and CD44 $4^{15,20}$.

Our previous studies have implied that forced activation of AMP-activated protein kinase (AMPK) can inhibit human glioma cells ${ }^{21,22}$. Thr-172 phosphorylation of AMPK $\alpha 1$ is essential for AMPK activation. AMPK activation inhibits mammalian target of rapamycin (mTOR) complex 1 (mTORC1), a key oncogenic cascade ${ }^{23,24}$. In human cancer cells, activated AMPK could also induce

\section{(c) The Author(s) 2019}

(c) (i) Open Access This article is licensed under a Creative Commons Attribution 4.0 International License, which permits use, sharing, adaptation, distribution and reproduction cc) in any medium or format, as long as you give appropriate credit to the original author(s) and the source, provide a link to the Creative Commons license, and indicate if changes were made. The images or other third party material in this article are included in the article's Creative Commons license, unless indicated otherwise in a credit line to the material. If material is not included in the article's Creative Commons license and your intended use is not permitted by statutory regulation or exceeds the permitted use, you will need to obtain permission directly from the copyright holder. To view a copy of this license, visit http://creativecommons.org/licenses/by/4.0/. 
growth inhibition and cell-cycle arrest by stabilizing and activating $\mathrm{p} 53^{25}$. Moreover, AMPK activation is shown to trigger autophagy and degradation of multiple growth factor receptors (i.e., epidermal growth factor receptor (EGFR) and platelet-derived growth factor receptor $\alpha$ (PDGFR $\alpha)$ ), thereby causing cancer cell inhibition ${ }^{26}$.

AMPK $\alpha 1$ expression is often sequestered in human cancer cells. Pineda et al. showed that MAGEA6-TRIM28 complex is a cancer-specific ubiquitin ligase, responsible for degradation AMPK $\alpha 1$ only in cancer cells ${ }^{27}$. We have previously shown that MAGEA6 knockdown by targeted short hairpin RNA (shRNA) restored AMPK $\alpha 1$ expression, causing glioma cell death and apoptosis ${ }^{21}$. In the present study, we will show that Lnc-THOR-IGF2BP1 cascade is essential for MAGEA6 expression in glioma cells. Inhibition of Lnc-THOR-IGF2BP1 cascade will induce MAGEA6 downregulation, AMPK $\alpha 1$ expression, and AMPK signaling activation, inhibiting glioma cell survival in vitro and in vivo.

\section{Materials and methods Chemicals and reagents}

Puromycin and neomycin were obtained from SigmaAldrich (St. Louis, MO). Cell culture reagents were provided by Gibco-BRL (Grand Island, NY). The antiMAGEA6 antibody (ab38495) was purchased from Abcam (Shanghai, China). All other antibodies were provided by Cell Signaling Tech (Shanghai, China). TRIzol reagents for RNA assays, Lipofectamine 2000, and other transfection reagents were obtained from Invitrogen (Shanghai, China).

\section{Cell culture}

Cultures of HCN-1a human neuronal cells, A172 and U251MG ("U251") human glioma cells, as well as the primary human astrocytes, were described earlier ${ }^{22,28}$. The human glioma cells, derived from two primary glioma patients, were provided by $\mathrm{Dr}$. $\mathrm{Cao}^{29,30}$, which were named as "Pri-1/Pri-2," and cultured as previously described $^{30,31}$. The protocols of studying human cells and tissues were approved by the Ethics Review Board of Shanghai Jiao-Tong University School of Medicine, according to Declaration of Helsinki.

\section{Human tissues}

As reported earlier ${ }^{21}$, a total of five glioma tissues, along with paired surrounding normal brain tissues, were acquired and stored in liquid nitrogen. Tissues were separated, thoroughly washed, minced, and homogenized by the tissue lysis buffer (BiYunTian, Wuxi, China). Written informed consent was obtained from each participant.

\section{Quantitative real-time reverse transcriptase polymerase chain reaction (qPCR)}

As reported ${ }^{21}, 500$ ng RNA of each sample was applied in the reverse transcription (RT) reaction with specific RT primers and superscript III reverse transcriptase (Invitrogen). Afterwards, $100 \mathrm{ng}$ obtained complementary DNA (cDNA) template was mixed with SYBR Master Mix (Applied Biosystem) and $200 \mathrm{nM}$ primers. We utilized ABI Prism 7600H Fast Real-Time PCR system for qPCR assays. The primers are listed in Table 1. qPCR quantification was through $2^{-\Delta \mathrm{Ct}}$ method using the following formula: $2^{- \text {(Ct of target gene }-\mathrm{Ct} \text { of reference gene) }}$. qPCR primers are listed in Table 1.

\section{Lnc-THOR shRNA}

A set of two shRNAs, against non-overlapping sequence of $L n c-T H O R$ ("Seq1/2," designed and verified by Genechem, Shanghai, China), were individually inserted into GV248 construct. The construct, along with the lentivirus package plasmids (Genechem), were transfected to HEK293 cells to generate $L n c$-THOR shRNA lentivirus. The virus was enriched, filtered, and added to glioma cells (plated at a density of $1 \times 10^{5}$ cells/well into 6 -well plates).

\section{Table 1 Primers utilized in this study}

\begin{tabular}{|c|c|c|}
\hline Gene name & Forward primers & Reverse primers \\
\hline GAPDH & 5'-CACCACCATGGAGAAGGCTGG-3' & 5'-GAAGTCAGAGGAGACCACCTG-3' \\
\hline MAGEA6 & 5'-TGGAGGACCAGAGGCCCCC-3' & 5'-CAGGATGATTATCAGGAAGCCTGT-3' \\
\hline LnC-THOR & 5'-CAAGGTGCTTCTCTCTGGATTT-3' & 5'-GCCAAAGTCATTTGTTGGGTAT-3' \\
\hline U6 & 5'-CTCGCTTCGGCAGCACATATACT-3' & 5'-ACGCTTCACGAATTTGCGTGTC-3' \\
\hline AMPKal & 5'-AGGAAGAATCCTGTGACAAGCAC-3' & 5'-CCGATCTCTGTGGAGTAGCAGT-3' \\
\hline Gli1 & 5'-AGCCTTCAGCAATGCCAGTGAC-3' & 5'-GTCAGGACCATGCACTGTCTTG-3' \\
\hline Myc & 5'-CCTGGTGCTCCATGAGGAGAC-3' & 5'-CAGACTCTGACCTTTTGCCAGG-3' \\
\hline IGF2 & 5'-TGGCATCGTTGAGGAGTGCTGT-3' & 5'-ACGGGGTATCTGGGGAAGTTGT-3 \\
\hline
\end{tabular}


Cells were then subjected to selection by using puromycin $(2.5 \mu \mathrm{g} / \mathrm{mL}$, for $10-12$ days). In stable cells, Lnc-THOR knockdown was verified by qPCR assay.

\section{Lnc-THOR KO}

The CRISPR/Cas9 Lnc-THOR KO construct (with sgRNA, 5'-CACCgAGGGTGTAGCGCGGGCTAGA-3', R: 5'-AAACTCTAGCCCGCGCTACACCCTC-3 ${ }^{15}$ ) was provided by Dr. Liang, which was transfected to glioma cells (plated at a density of $1 \times 10^{5}$ cells/well into 6-well plates) by Lipofectamine 2000 reagents. Fluorescent-activated cell sorting (FACS)-mediated sorting of the green fluorescent protein (GFP)-positive cells were performed to select monoclonal cells, which were then cultured in the puromycin-containing complete medium to achieve stable cells. $L n c-T H O R$ KO was verified by qPCR assay.

\section{Lnc-THOR overexpression}

The full-length $L n c-T H O R$ was amplified by the described primers ${ }^{15}$ and inserted to the GV248 lentiviral construct (Genechem). The lentiviral GV248-Lnc-THOR construct ("LV-Lnc-THOR") was transfected to glioma cells (plated at a density of $1 \times 10^{5}$ cells/well into 6-well plates), followed by selection using puromycin $(2.5 \mu \mathrm{g} /$ $\mathrm{mL}$ ) for 10-12 days. In stable cells, Lnc-THOR overexpression was verified by qPCR assay.

\section{Cell viability assay}

Briefly, cells were plated at a density of $3 \times 10^{3}$ cells/well into 96-well plates. Following culture of $96 \mathrm{~h}, 3-[4,5-$ dimethylthiazol-2-yl]-2,5 diphenyl tetrazolium bromide (MTT; $5 \mathrm{mg} / \mathrm{mL}, 20 \mu \mathrm{L} /$ well, dissolved in phosphatebuffered saline (PBS)) was added, cells were further incubated for additional $2 \mathrm{~h}$, and its optical density (OD) was tested at $590 \mathrm{~nm}$.

\section{Cell proliferation assays}

For the soft agar colony-formation assay, A172 cells (5000 cells of each treatment) were re-suspended in agar (0.5\%)-containing complete medium (with fetal bovine serum (FBS)) and added on the top of $10-\mathrm{cm}$ culture dishes. After incubation for 10 days, A172 cell colonies were stained and manually counted. The detailed protocol for the 5-ethynyl-2'-deoxyuridine (EdU) staining assay was reported earlier ${ }^{32}$.

\section{Apoptosis assays}

The detailed protocols of apoptosis assays, including Histone DNA enzyme-linked immunosorbent assay and Annexin V FACS, as well as terminal deoxynucleotidyl transferase-mediated dUTP-fluorescein nick end labeling (TUNEL) staining assay and caspase-3/caspase-9 activity assays, were described in previous studies ${ }^{33,34}$.

\section{"Transwell" in vitro migration assay}

A172 glioma cells $\left(3 \times 10^{5}\right.$ cells in $300 \mu \mathrm{L}$ medium) were seeded into the upper part of the "Transwell" chambers (12- $\mu \mathrm{m}$ pore size, BD Biosciences). The lower compartments were filled with complete medium with $10 \%$ FBS. After $48 \mathrm{~h}$, on the upper surfaces the non-migrated A172 cells were removed. On the lower surfaces, the migrated cells were fixed, stained, and counted.

\section{Western blotting analysis}

The detail protocol of western blotting assay was described in our previous studies ${ }^{9,10}$. Briefly, for each treatment $40 \mu \mathrm{g}$ of protein lysates (in each lane) were separated in denaturing $10-12 \%$ polyacrylamide gels and transferred to a polyvinylidene difluoride blots. After blocking (in 10\% milk PBST solution) and three washes in TBST, blots were incubated with the indicated primary and secondary antibodies. Immuno-reactive proteins were detected by an enhanced chemiluminescence kit (Amersham, Shanghai, China) and analyzed through autoradiography. ImageJ software $(\mathrm{NIH})$ was utilized for the quantification of the protein band, which was always normalized to the loading control.

\section{AMPKa1 shRNA}

As described ${ }^{21}$, the lentiviral AMPK $\alpha 1$ shRNA was added to A172 cells (plated at a density of $1 \times 10^{5}$ cells/ well into 6-well plates) for $48 \mathrm{~h}$. Puromycin $(2.5 \mu \mathrm{g} / \mathrm{mL})$ containing complete medium was added to select stable cells for 5-6 days. Control cells were infected with the lentiviral scramble control shRNA ("sh-C"). AMPK $\alpha 1$ silencing in the stable cells was confirmed by western blotting.

\section{AMPKa1 dominant-negative mutation}

The dominant-negative AMPK $\alpha 1$ (dnAMPK $\alpha 1$, T172A, as reported ${ }^{21}$ ) or the empty vector (pSuper-neo-Flag) was transfected to A172 cells (plated at a density of $1 \times 10^{5}$ cells/well into 6-well plates) by Lipofectamine 2000 . Neomycin $(1.0 \mu \mathrm{g} / \mathrm{mL})$ was added to select stable cells for 5-6 days. Expression of the mutant AMPK $\alpha 1$ was verified by western blotting.

\section{AMPK activity assay}

Following the treatments, $200 \mu \mathrm{g}$ of total cellular lysates were first incubated with anti-AMPK $\alpha 1$ antibody. The AMPK activity was examined in the kinase assay buffer by adding AMP- $\left[\gamma_{-}{ }^{32} \mathrm{P}\right]$ ATP mixture and AMPK substrate SAMS (HMRSAMSGLHLVKRR) peptide ${ }^{35}$. Phosphocellulose paper was added afterwards, stopping the reactions. The AMPK radioactivity was examined by a scintillation counter, and its value was normalized to control level. 


\section{IGF2BP1 or AMPKa1 KO}

A172 cells were seeded onto 6-well tissue culture plates at a density of $1 \times 10^{5}$ cells/well. The lenti-CRISPR/Cas9IGF2BP1-KO-GFP construct (provided by Dr. Zhao ${ }^{36}$ ) or the lenti-CRISPR/Cas9-AMPK $\alpha 1-K O-G F P$ construct (from Dr. $\mathrm{Li}^{37}$ ) was transfected to A172 cells through Lipofectamine 2000 protocol. FACS-mediated sorting of the GFP-positive cells were performed to select the monoclonal cells, which were then cultured in the puromycin-containing complete medium to achieve stable cells. IGF2BP1 or AMPK $\alpha 1 \mathrm{KO}$ in the stable cells was confirmed by western blotting and/or qPCR assays.

\section{Ectopic IGF2BP1 overexpression}

The recombinant adenovirus encoding IGF2BP1 expression construct (provided by Dr. Zhao ${ }^{36}$ ) was added to cultured A172 cells (plated at a density of $1 \times 10^{5}$ cells/ well into 6-well plates) for $48 \mathrm{~h}$. Cells were thereafter subjected to puromycin $(2.5 \mu \mathrm{g} / \mathrm{mL})$ selection for another 5-6 days. IGF2BP1 overexpression was confirmed by western blotting.

\section{RNA immunoprecipitation (RIP)}

RIP experiments were carried out through a described protocol $^{38}$. Briefly, glioma cells were trypsinized, washed, and incubated with $0.3 \%$ formaldehyde and glycine ${ }^{39}$. Afterwards, glioma cells were washed, and resuspended, with the pellets dissolved in the RIP buffer ${ }^{38}$. The lysates were then incubated with the anti-IGF2BP1 antibody. Pellets were washed, re-suspended, and incubated with proteinase K-containing buffer. IGF2BP1-bound LncTHOR and MAGEA6 mRNA was tested by qPCR, with its level normalized to internal controls.

\section{RNA Pull-Down assay}

RNA Pull-Down was carried out using a previously described protocol ${ }^{39}$. In short, the biotin-labeled fulllength Lnc-THOR (provided by Dr. Wang ${ }^{39}$ ) was folded in RNA structure buffer and incubated with cleared nuclei lysates of the glioma cells together with Dynabeads MyOne Streptavidin C1 magnetic beads ("Beads," again provided by Dr. Wang ${ }^{39}$ ). Beads were washed, with the retrieved proteins examined by western blotting.

\section{Xenograft assay}

As reported ${ }^{21}$, the female severe combined immunodeficient (SCID) mice were housed under standard procedures. Lnc-THOR shRNA-bearing stable A172 cells, Lnc-THOR KO stable A172 cells, or the parental control A172 cells $\left(5 \times 10^{6}\right.$ cells in $200 \mu \mathrm{l}$ of Matrigel gel, no serum, each mouse) were subcutaneously (s.c.) injected to the flanks. When the volume reached approximately $100 \mathrm{~mm}^{3}$ for each tumor ("Day-0"), the recordings were started. Tumor volumes were calculated as described ${ }^{21}$.
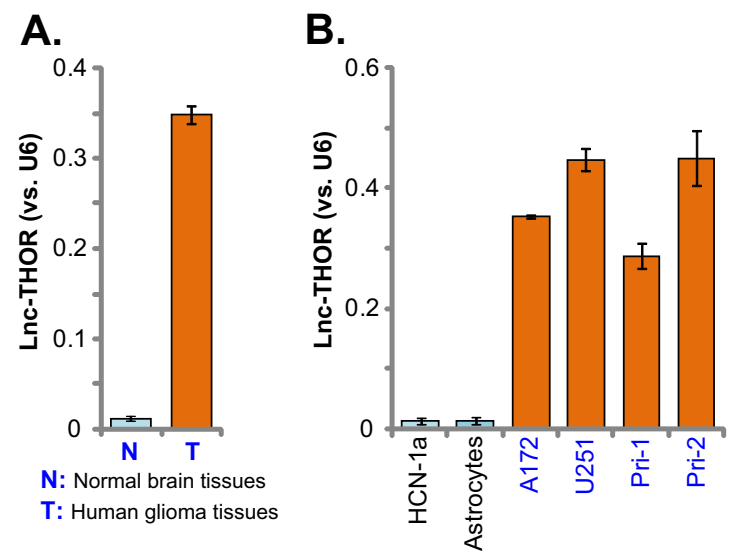

Fig. 1 LnC-THOR expression in human glioma tissues and cells. Lnc-THOR expression levels in human glioma tissues ("T," $n=5$ ) and paired surrounding normal brain tissues ("N," $n=5$ ) were tested by quantitative real-time PCR (qPCR) (a). The primary human astrocytes ("Astrocytes"), HCN-1a neuronal cells, as well as A172 and U251MG ("U251") glioma cell lines and primary human glioma cells ("Pri-1/ Pri-2"), were subjected to qPCR assay of Lnc-THOR expression (b). Data are presented as mean $\pm \mathrm{SD}(n=5)$. Experiments in this figure were repeated three times, and similar results were obtained

All animal procedures were approved by IACUC of Shanghai Jiao-Tong University School of Medicine.

\section{Statistical analysis}

All statistics were calculated by using the SPSS 18.0 statistical software (SPSS, Chicago, IL). Descriptive statistics including mean and standard deviation (SD) along with one-way analyses of variance were applied to determine significant differences. Two-tailed unpaired $T$ test (Excel 2013) was applied to test significance between the two treatment groups. $p<0.05$ was considered significant.

\section{Results}

\section{Lnc-THOR expression in human glioma tissues and cells}

First, we tested the expression of Lnc-THOR in human glioma tissues. As described in our previous studies ${ }^{21}$, a total of five pairs of human glioma tissues ("T") and paired surrounding normal brain tissues ("N") were analyzed, and GPCR assay results in Fig. 1a show that Lnc-THOR levels are high in human glioma tissues, whereas its levels in normal brain tissues are, however, extremely low (Fig. 1a). Further studies show that LncTHOR is expressed in established (A172 and U251 lines) and primary human glioma cells (derived from two different patients, "Pri-1/-2") (Fig. 1b). Its expression is almost undetected in the primary human astrocytes ${ }^{22}$ and $\mathrm{HCN}-1 \mathrm{a}$ neuronal cells ${ }^{22}$ (Fig. 1b). These results confirm unique $L n c-T H O R$ expression in human glioma tissues and cells. 

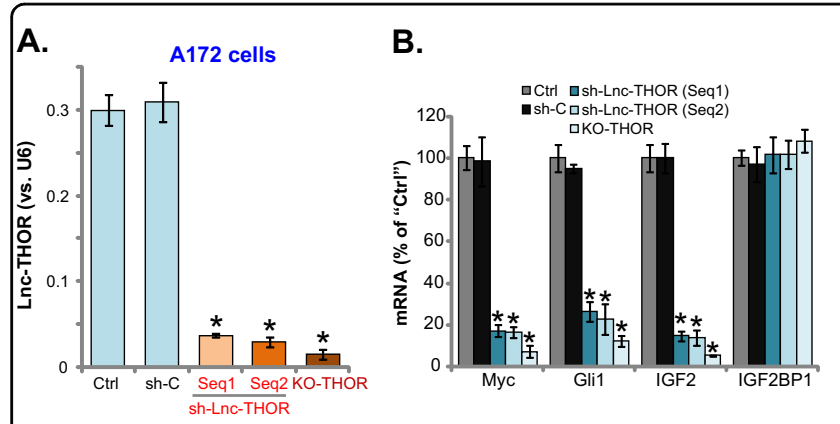

E.
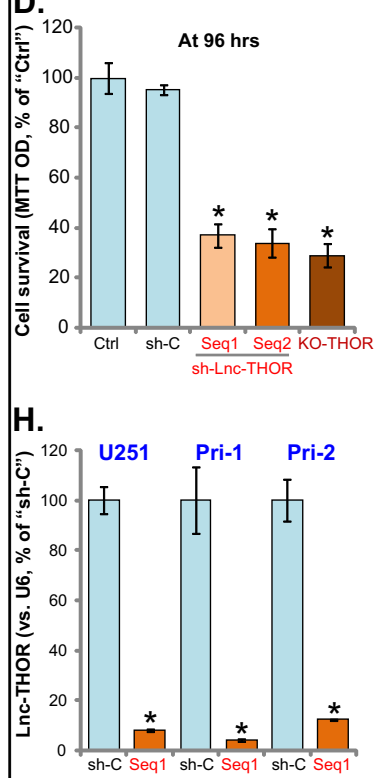
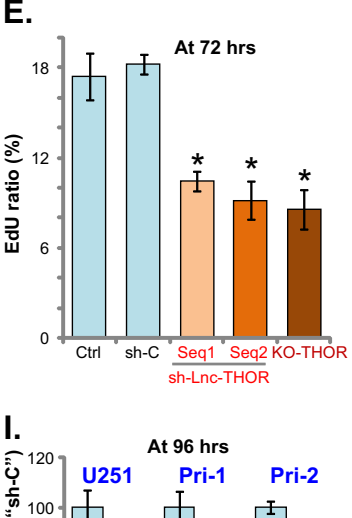

F.

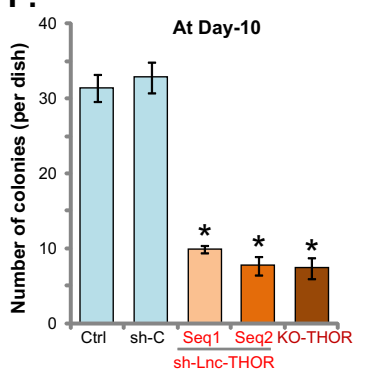

J.

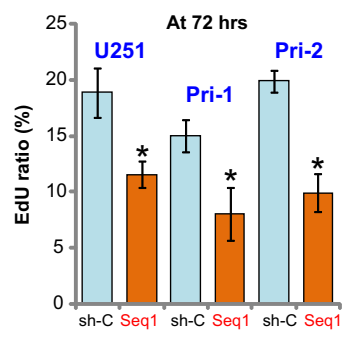

C.

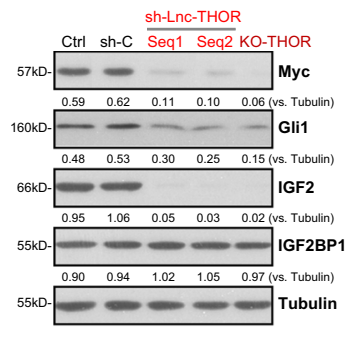

G.

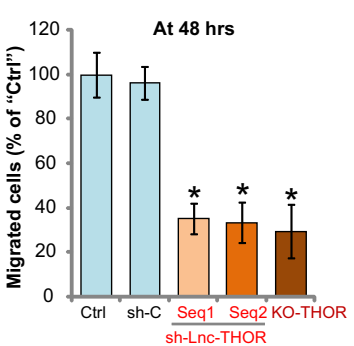

K.

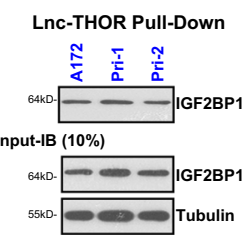

L.

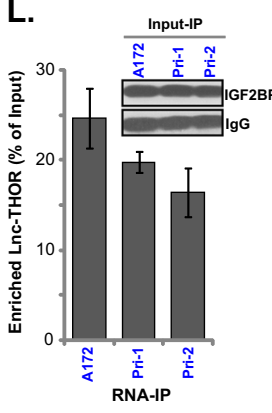

Fig. 2 Lnc-THOR silencing or KO inhibits human glioma cell survival and proliferation. The genetically modified stable A172 cells, with LncTHOR shRNA ("sh-Lnc-THOR," with non-overlapping sequences, "Seq1/2"), scramble non-sense control shRNA ("sh-C"), or the lenti-CRISPR/Cas9 LncTHOR-KO construct ("KO-THOR"), were established, the expression of Lnc-THOR and listed genes in the stable cells and in parental control A172 cells ("Ctrl") were tested by qPCR and western blotting assays (a-c); Cells were further cultured for the indicated time, and cell viability was tested by MTT assay (d); Cell proliferation was analyzed by EdU staining (e) and soft agar colony-formation ( $\mathbf{f}$ ) assays; Cell migration was tested by "Transwell" assays (g). U251MG ("U251") and primary human glioma cells ("Pri-1/Pri-2") were transfected with lentiviral Lnc-THOR shRNA ("sh-Lnc-THOR," "Seq1") or "sh-C" and stable cells were established via puromycin selection. Lnc-THOR levels were tested by qPCR assay (h); Cell survival and proliferation were tested by MTT assay (i) and EdU staining assay (j), respectively. Western blotting assay of the IGF2BP1 protein retrieved by in vitro-transcribed Lnc-THOR in A172 cells and primary human glioma cells (k). QPCR analyses of Lnc-THOR expression enriched by the IGF2BP1 protein in A172 cells and primary human glioma cells (I). For all the in vitro function assays, the exact same amount of viable cells was initially seeded in each well/dish ("0 $\mathrm{h}$ ") (same for all figures). Blot data were quantified and normalized to the corresponding loading control (c). Data are presented as mean \pm SD $(n=5)$. ${ }^{*} p<0.05$ vs. "sh-C" cells. Experiments in this figure were repeated three times, and similar results were obtained

\section{Lnc-THOR silencing or KO inhibits human glioma cell progression in vitro}

In order to study the function of $L n c-T H O R$ in human glioma cells, two lentivirus-encoded $L n c-T H O R$ shRNAs, with non-overlapping sequences ("Seq $1 / 2$ "), were individually transfected to A172 glioma cells. Following puromycin selection, the stable cells were established ("shLnc-THOR" cells). Moreover, the lenti-CRISPR/Cas9 Lnc-THOR-KO construct (see "Methods" section) was transfected to A172 cells. Stable cells ("KO-THOR" cells) were established by FACS sorting of GFP cells and puromycin selection. Analyzing Lnc-THOR expression in the stable cells, by qPCR, confirmed that $L n c$-THOR levels were dramatically downregulated in the stable cells with Lnc-THOR shRNA or Lnc-THOR KO construct (Fig. 2a). Lnc-THOR binds to IGF2BP1 to ensure mRNA stabilization of key pro-cancerous genes, including IGF2, Gli1, and $M y c^{15,16,18,20}$. In A172 glioma cells, mRNA levels of $I G F 2$, Gli1, and Myc were significantly downregulated in LncTHOR-silenced or Lnc-THOR-KO A172 cells (Fig. 2b). IGF2, Gli1, and Myc proteins were downregulated as well (Fig. 2c). Lnc-THOR shRNA or KO did not affect 
IGF2BP1 mRNA (Fig. 2b) and protein expression (Fig. 2c). The scramble non-sense control shRNA ("sh-C") had no significant effect on the expression of Lnc-THORIGF2BP1 pathway genes (Fig. 2a-c).

MTT, EdU staining, and soft agar colony-formation assays were performed to test glioma cell functions. When compared to control A172 cells, in Lnc-THOR-silenced or Lnc-THOR-KO A172 cells, MTT OD values (Fig. 2d), EdU percentages (Fig. 2e), and the number of colonies (Fig. 2f) were significantly decreased. A172 cell in vitro migration, tested by the "Transwell" assays, were significantly inhibited by $L n c-T H O R$ shRNA or KO (Fig. $2 \mathrm{~g}$ ). In U251MG cells and primary human glioma cells ("Pri-1/2"), transfection of the lentiviral $L n c-T H O R$ shRNA ("Seq1") induced $>90 \%$ reduction of $L n c-T H O R$ expression (Fig. 2h), causing reduced MTT OD (Fig. 2i) and EdU ratio (Fig. 2j). These results show that Lnc-THOR silencing or $\mathrm{KO}$ inhibits glioma cell growth and migration.

Lnc-THOR-IGF2BP1 binding has been reported in other cancer cells ${ }^{15,20,39}$. To test the direct association between $L n c-T H O R$ and the IGF2BP1 protein in glioma cells, we employed a $L n c-T H O R$ pull-down assay ${ }^{39}$. Results demonstrated that the IGF2BP1 protein is co-precipitated with the in vitro-transcribed biotinylated Lnc-THOR (provided by Dr. Wang ${ }^{39}$ ) in both A172 cells and primary human glioma cells ("Pri-1/-2") (Fig. 2k). In addition, the RIP assay results show again the direct binding between $L n c$-THOR and the IGF2BP1 protein in A172 cells and the primary human glioma cells (Fig. 2l).

\section{Lnc-THOR silencing or KO induces apoptosis activation in human glioma cells}

The potential effect of $L n c-T H O R$ on glioma cell apoptosis was studied. As shown, in the A172 cells with $L n c$ THOR shRNA ("Seq-1/-2") or Lnc-THOR-KO construct ("KO-THOR" cells, see Fig. 2), the activities of caspase-3 and caspase- 9 were significantly increased (compared to control A172 cells, Fig. 3a). Furthermore, Lnc-THOR silencing or $\mathrm{KO}$ in A172 cells induced cleavages of caspase-3, caspase-9, and PARP (poly ADP-ribose polymerase) (Fig. 3b), as well as accumulation of histonebound DNA (Fig. 3c). In addition, Annexin V percentages (Fig. 3d, e) and nuclear TUNEL ratios (Fig. 3f) were significantly increased in $L n c-T H O R$-silenced or -KO cells. In U251MG and primary human glioma cells ("Pri-1/-2"), the lentiviral Lnc-THOR shRNA ("Seq1") similarly induced increases of nuclear TUNEL ratios (Fig. 3g). Taken together, these results clearly show that $L n c-T H O R$ silencing or $\mathrm{KO}$ provokes apoptosis activation in human glioma cells.

\section{Lnc-THOR overexpression promotes human glioma cell survival and proliferation}

Since Lnc-THOR silencing or KO inhibited glioma cell growth and migration, we hypothesized that forced overexpression of $L n c-T H O R$ shall exert opposite functions. To test this hypothesis, the lentivirus encoding $L n C$ THOR expression construct (see "Methods") was transduced to A172 glioma cells. Following selection using the puromycin-containing medium, two A172 cell lines were established ("Line1/2"). Testing Lnc-THOR expression, by qPCR, confirmed that $L n c-T H O R$ levels were significantly increased in the stable cells with $L n c-T H O R$ construct ("OE-Lnc-THOR" cells). Consequently, mRNA and protein expression of IGF2BP1 targets, IGF2, Gli1, and $M y c$, were upregulated (Fig. 4b, c). IGF2BP1 expression was again not changed (Fig. 4b, c). As compared to vector control cells, increased MTT OD values (Fig. 4d), EdU staining (Fig. 4e), and colony formation (Fig. 4f) were detected in the OE-Lnc-THOR cells. These results indicate that $L n c-T H O R$ overexpression promotes A172 cell growth and migration. Similarly in U251MG cells and primary human glioma cells ("Pri-1/-2"), adding $L n c$ THOR-expressing lentivirus increased Lnc-THOR expression (Fig. 4g), enhancing cell survival (Fig. 4h) and proliferation (Fig. 4i).

\section{Lnc-THOR depletion activates MAGEA6-AMPK signaling in glioma cells}

MAGEA3/6-TRIM28 complex is a cancer-specific ubiquitin ligase of AMPK $\alpha 1^{21,27,40,41}$. Our previous study has shown that MAGEA6 sequesters AMPK $\alpha 1$ in glioma cells, causing mTORC1 overactivation and cancer cell growth. Reversely, MAGEA6 silencing inhibits human glioma cell cells via re-activation of AMPK signaling ${ }^{21}$. RIP assays (same experiments as Fig. 2l) confirmed the direct binding between MAGEA6 $m R N A$ and the IGF2BP1 protein in A172 cells and the primary human glioma cells (Fig. 5a). Importantly, Lnc-THOR silencing (by "Seq1" shRNA, see Fig. 2) or $\mathrm{KO}$ (see Fig. 2) downregulated MAGEA6 mRNA (Fig. 5b) and protein (Fig. 5c) in A172 cells. Consequently, AMPK $\alpha 1$ protein expression and AMPK activation (AMPK $\alpha 1-A C C$ phosphorylation) were significantly increased (Fig. 5c). AMPK activity was increased as well in Lnc-THOR-silenced/-KO cells (Fig. 5c).

Activated AMPK will inhibit human cancer cells via regulating its downstream effectors, causing mTORC1 inhibition $^{23,24,42}$, autophagy induction ${ }^{42-44}$, and receptor tyrosine kinase (RTK) degradation ${ }^{10,26}$. In A172 cells, LncTHOR silencing or KO largely inhibited p70S6K1-S6 phosphorylation, indicating mTORC1 inhibition (Fig. 5d). RTKs, including EGFR and PDGFR $\alpha$, were downregulated (Fig. 5e). Significantly, AMPKa1 mRNA levels were unchanged by Lnc-THOR silencing or KO (Fig. 5f). Based on these results, we propose that $L n c$-THOR depletion activates AMPK activation possibly by downregulating AMPKa1's ubiquitin ligase MAGEA6.

To test whether AMPK activation mediated Lnc-THORdepletion-induced cytotoxicity of glioma cells, we utilized 


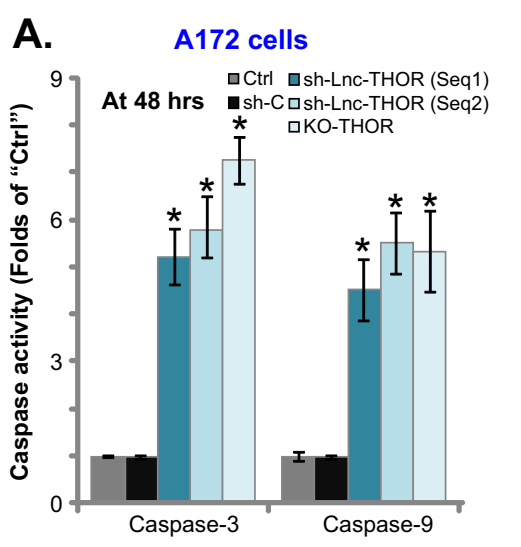

B.

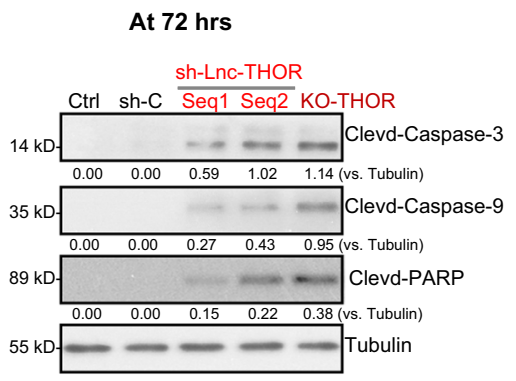

C.

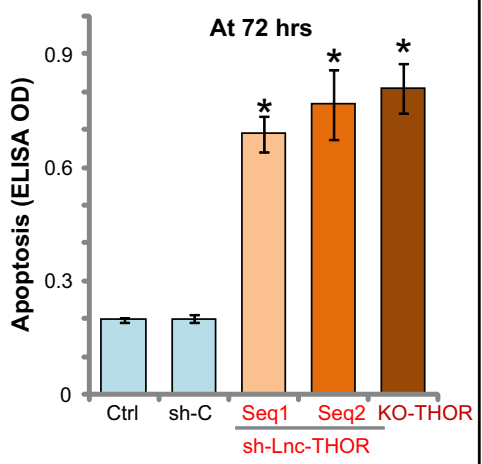

D.

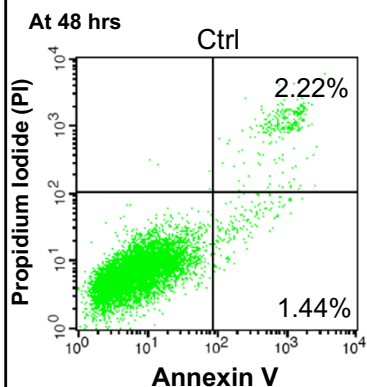

Annexin V

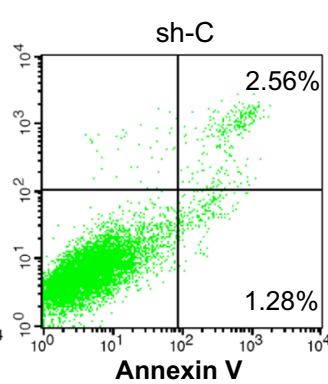

Annexin V

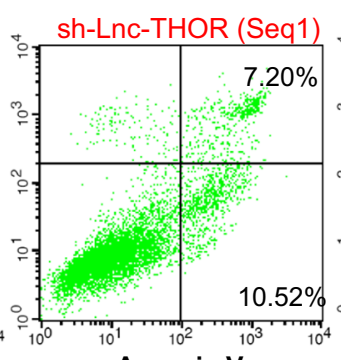

Annexin V

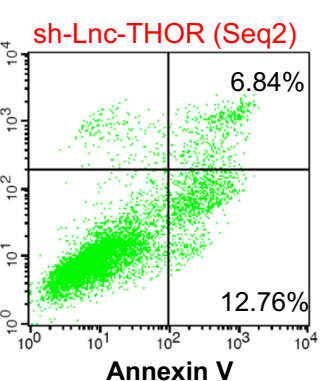

Annexin V

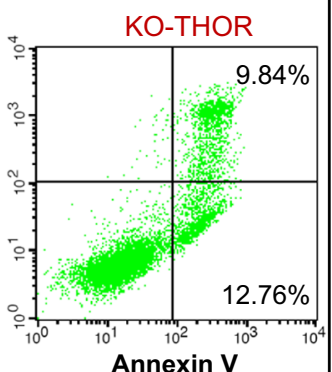

E.

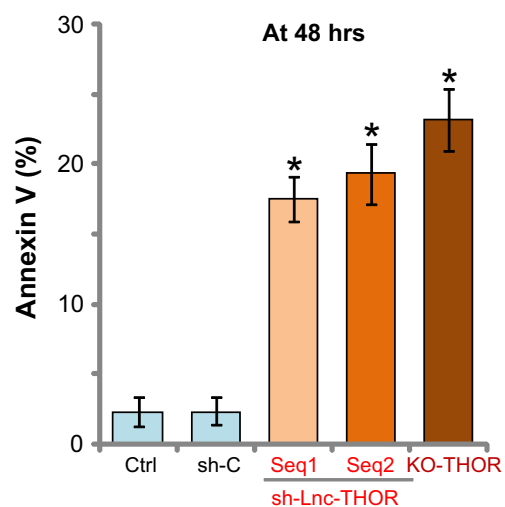

$\mathbf{F}$

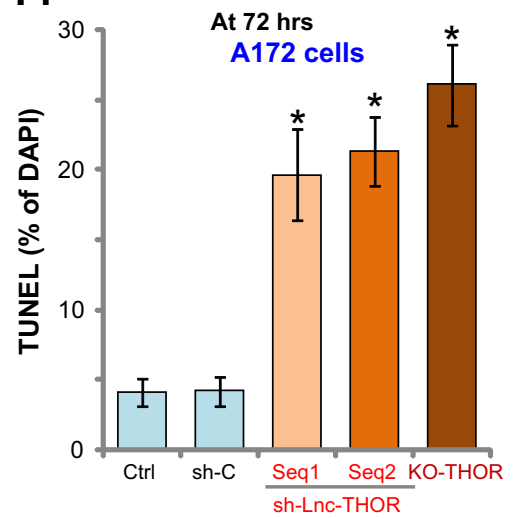

G.

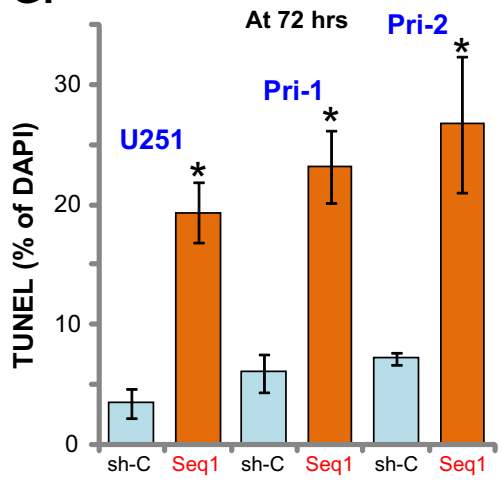

Fig. 3 Lnc-THOR silencing or KO induces apoptosis activation in human glioma cells. The genetically modified stable A172 cells, with $L$ LC-THOR shRNA ("sh-LnC-THOR," with non-overlapping sequences, "Seq1/2"), scramble non-sense control shRNA ("sh-C"), or the lenti-CRISPR/Cas9 Lnc-THOR$\mathrm{KO}$ construct ("KO-THOR"), were established; caspase-3/-9 activity and apoptosis activation in the stable cells and in parental control A172 cells ("Ctrl") were tested by the listed assays (a-f). U251MG ("U251") and primary human glioma cells ("Pri-1/Pri-2") were transfected with Lnc-THOR shRNA ("shLnc-THOR," "Seq1") or "sh-C," stable cells were established via puromycin selection, and cell apoptosis was quantified by the TUNEL staining (g). Blot data were quantified and normalized to the corresponding loading control (b). Data were presented as mean \pm SD ( $n=5$ ). ${ }^{*} p<0.05$ vs. "sh-C" cells. Experiments in this figure were repeated three times, and similar results were obtained

previously described genetic strategies ${ }^{21}$ to block AMPK activation. The lentiviral AMPK $\alpha 1$ shRNA, the dominantnegative AMPK $\alpha 1$ ("dnAMPK $\alpha 1$," T172A) construct, or the lenti-CRISPR/Cas9 AMPK 1 KO construct was separately transduced to A172 cells. Stable cells were established via selection (see "Methods"). As shown, LncTHOR shRNA ("Seq1," see Fig. 2) induced AMPK activation or AMPK $\alpha 1-\mathrm{ACC}$ phosphorylation was almost completely blocked by AMPK $\alpha 1$ shRNA, dominantnegative mutation, and $\mathrm{KO}$ (Fig. $5 \mathrm{~g}$ ). As a result, $\mathrm{Lnc}$ THOR shRNA-induced glioma cell death (Fig. 5h) and apoptosis (Fig. 5i) were largely ameliorated. Therefore, AMPK activation mediates Lnc-THOR-depletion-induced glioma cell death. 


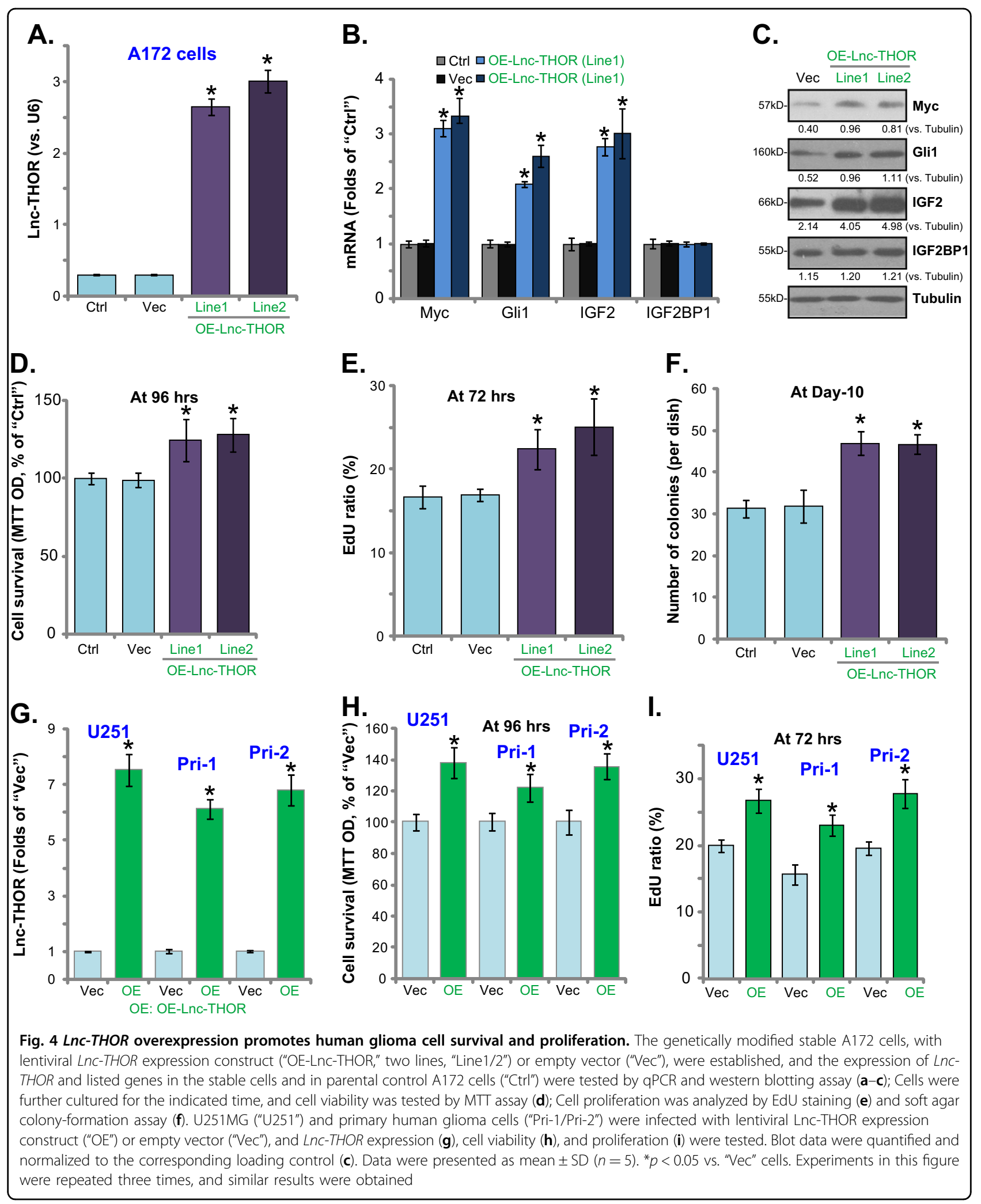




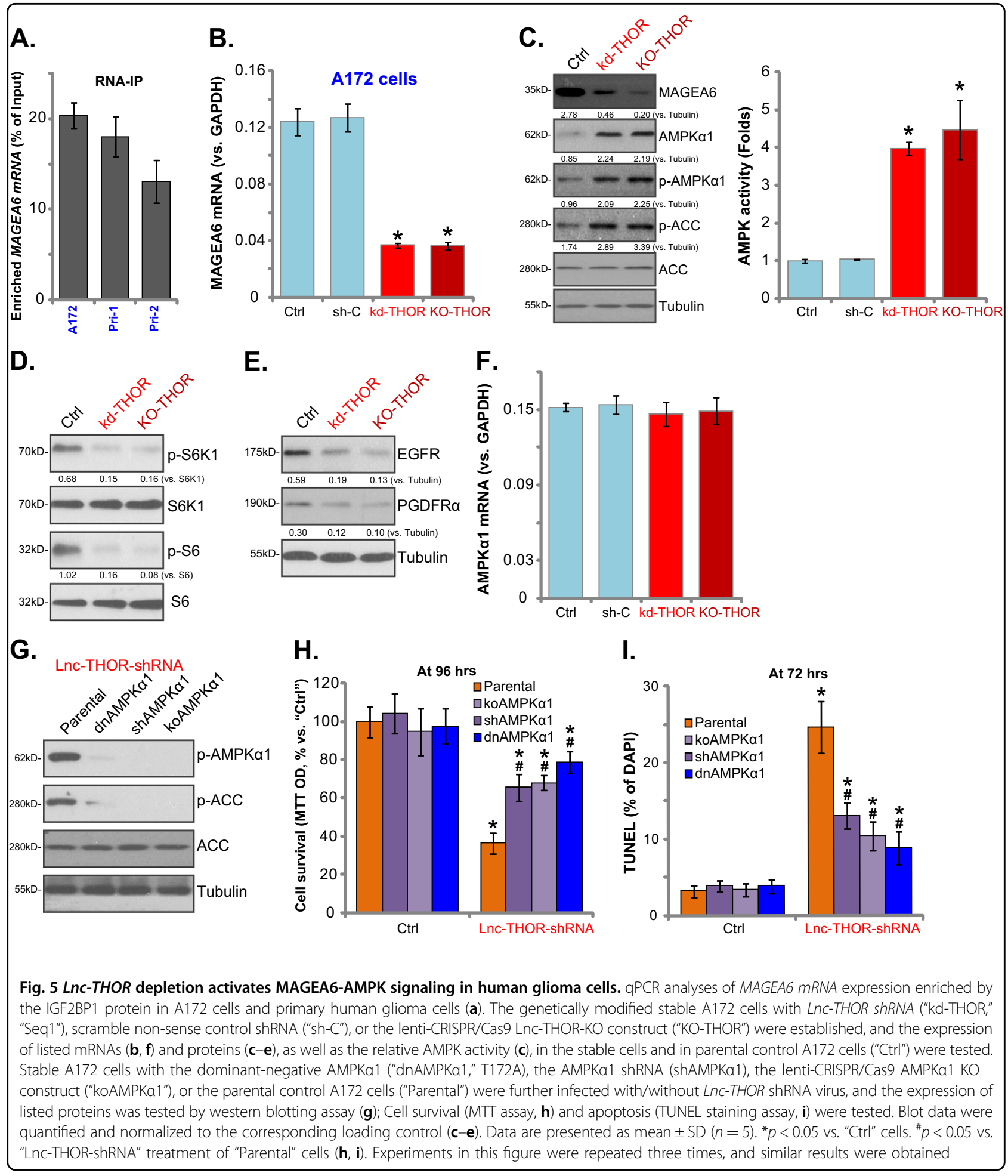

\section{Lnc-THOR silencing is ineffective in IGF2BP1-KO glioma} cells

Using the CRISPR/Cas9 gene-editing method (see ref. ${ }^{36}$ ), we established two lines of IGF2BP1 KO A172 cells (IGF2BP1 KO, "L1/L2"). qPCR results in Fig. 6a confirmed IGF2BP1 mRNA depletion, which did not affect the $L n c-T H O R$ expression (Fig. 6b). Importantly, in IGF2BP1 KO A172 cells, MAGEA6 mRNA (Fig. 6c) and protein (Fig. 6d) levels were significantly downregulated, accompanied with increased AMPK $\alpha 1$ expression (Fig. 6d) and AMPKo1-ACC phosphorylation (Fig. 6d), as well as increased AMPK activity (Fig. 6e). Therefore, IGF2BP1 is 


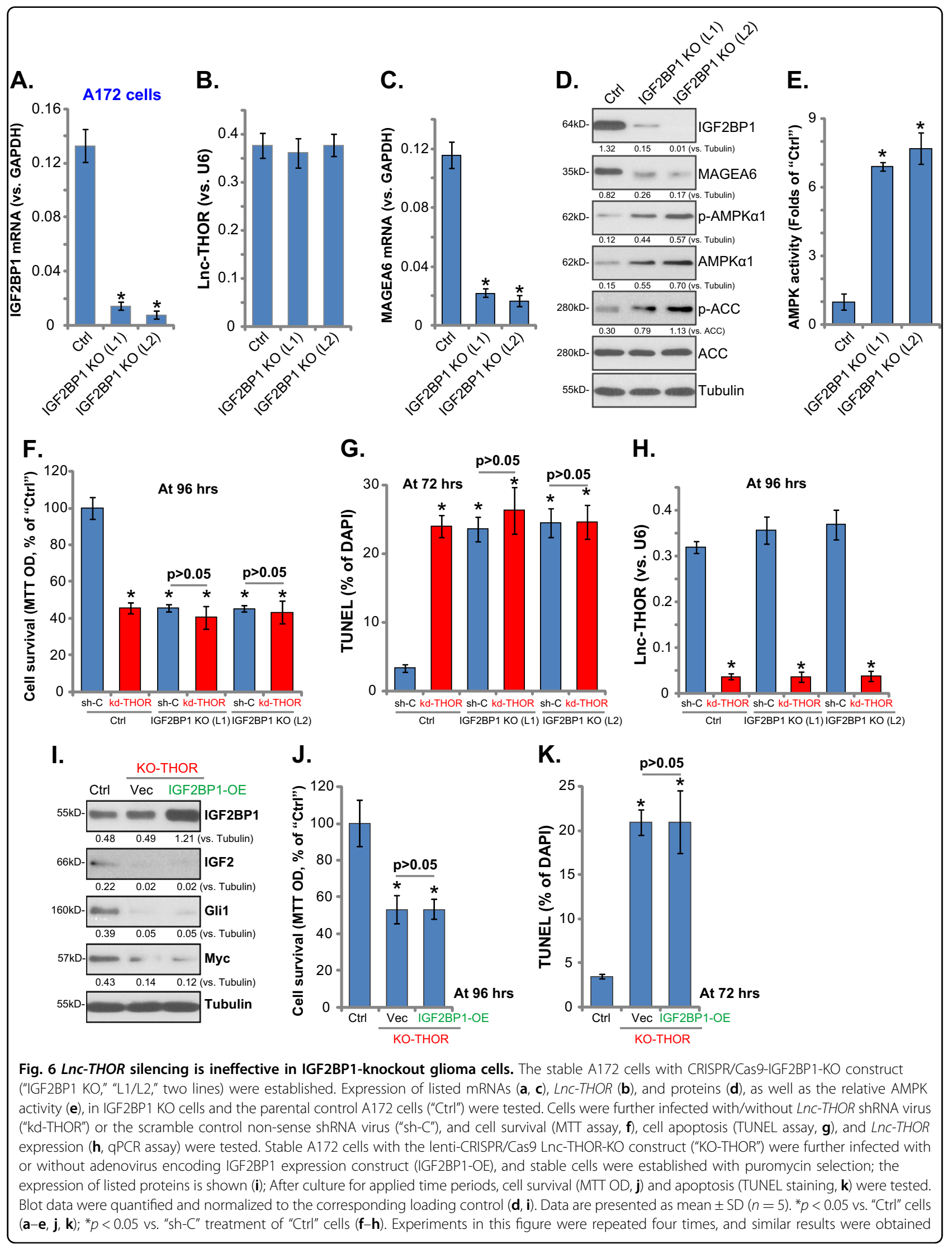




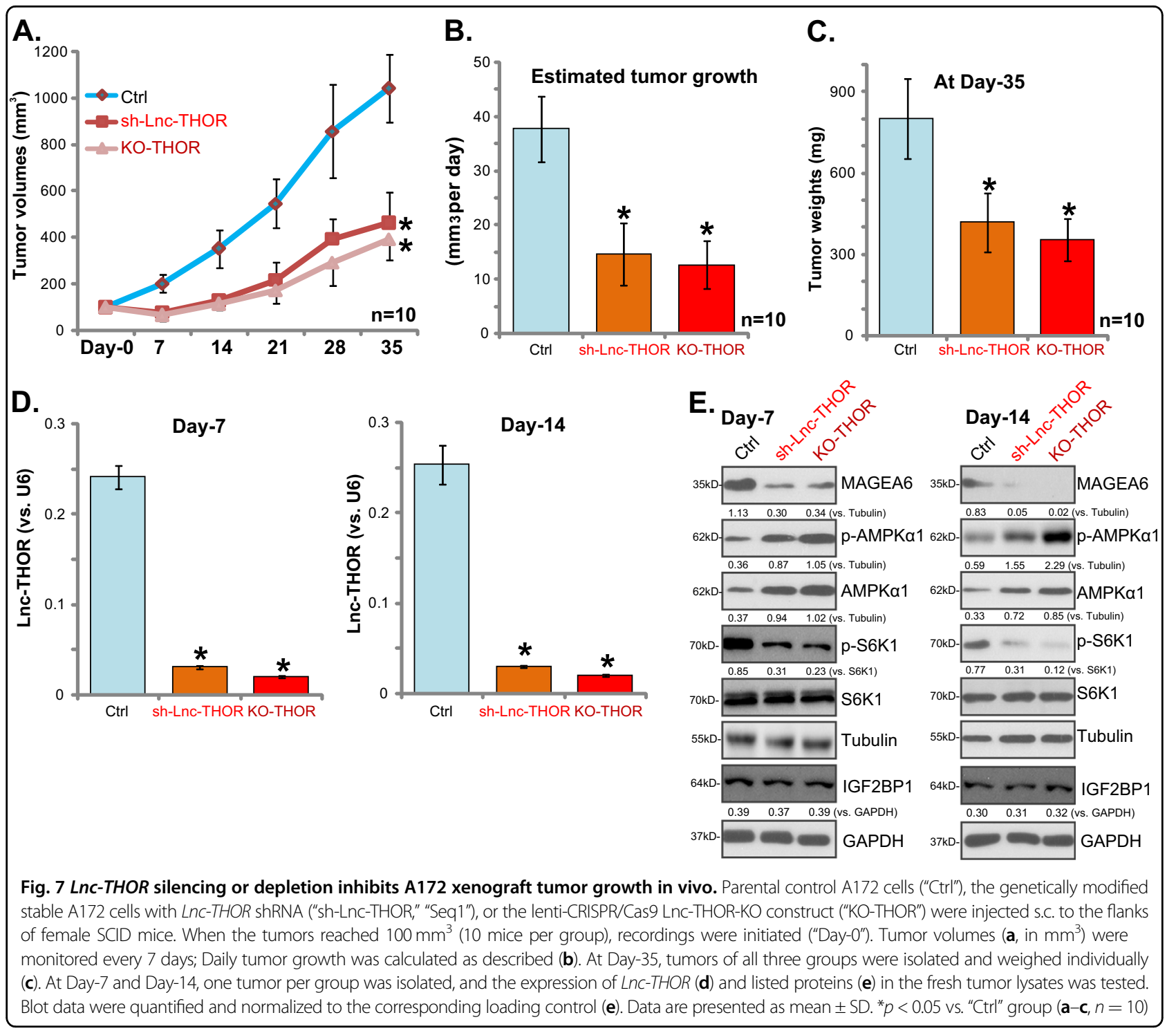

important for MAGEA6 expression and AMPK inactivation in glioma cells.

Similar to the phenotypes of $L n c-T H O R$ depletion, IGF2BP1 KO also promoted A172 cell death (Fig. 6f) and apoptosis (Fig. 6g). Importantly, adding Lnc-THOR shRNA lentivirus ("Seq1") was invalid in IGF2BP1 KO cells (Fig. 6f, g), although the applied shRNA did downregulate Lnc-THOR in A172 cells (Fig. 6h). These results confirm that $L n c$-THOR silencing is ineffective in IGF2BP1-KO glioma cells. Further studies demonstrated that Lnc-THOR KO (see Fig. 2) downregulated IGF2BP1's targets: IGF2, Gli1, and Myc, in A172 cells, which was not affected by ectopic IGF2BP1 overexpression (Fig. 6i). Furthermore, IGF2BP1 overexpression failed to reverse $L n c-T H O R$ KO-induced viability reduction (Fig. 6j) and apoptosis activation (Fig. 6k) in A172 cells.

\section{Lnc-THOR silencing or depletion inhibits A172 xenograft tumor growth in vivo}

As described in our previous study ${ }^{21}$, an A172 tumor xenograft SCID mice model was established to study the potential activity of $L n c-T H O R$ in vivo. The genetically modified stable A172 cells with Lnc-THOR shRNA ("shLnc-THOR," "Seq-1") or lenti-CRISPR/Cas9 Lnc-THORKO construct ("KO-THOR"), as well as the parental control A172 cells ("Ctrl"), were inoculated via s.c. injection to the flanks of the SCID mice. Tumor volumes were recorded, and the tumor growth curve results in Fig. 7a demonstrated that A172 tumor growth was significantly inhibited with Lnc-THOR silencing or depletion. Estimated daily tumor growth, calculated by (estimated tumor volume at Day35 - estimated tumor volume at Day-0)/35), was also significantly decreased in "sh-Lnc-THOR" tumors and "KOTHOR" tumors (Fig. 7b). Moreover, "sh-Lnc-THOR" 
tumors and "KO-THOR" tumors weighed significantly lower than "Ctrl" tumors (Fig. 7c). The body weights of the SCID mice were not significantly different between the three groups (data not shown). These results confirmed that Lnc-THOR silencing or depletion inhibited A172 xenograft tumor growth in vivo.

In order to test signaling changes in vivo, at Day-7 and Day-14, one tumor per group was isolated (total six tumors). Fresh tumor lysates were achieved and tested. When compared to "Ctrl" tumors, Lnc-THOR levels were significantly decreased in the "sh-Lnc-THOR" tumors and "KO-THOR" tumors (Fig. 7d), where MAGEA6 downregulation, AMPK $\alpha 1$ upregulation, and AMPK activation, as well as p-S6K1 inhibition, were detected (Fig. 7e). Total S6K1 and IGF2BP1 expression was unaffected by LncTHOR silencing or KO in tumor lysates (Fig. 7e). These results in vivo are therefore in line with the in vitro findings.

\section{Discussion}

The results of the current study indicate that $L n c-T H O R$ could possibly be a novel and important therapeutic target of human glioma. $L n c-T H O R$ is uniquely expressed in human glioma tissues and cells. Its expression is extremely low or even undetected in normal brain tissues, as well as in normal neuronal cells/astrocytes. In established (A172 cell line) and primary human glioma cells, Lnc-THOR shRNA or KO potently inhibited cell survival and proliferation, while provoking cell apoptosis. Contrarily, forced overexpression of Lnc-THOR can further promote glioma cell growth and migration. In vivo, A172 xenograft tumors with $L n c-T H O R$ silencing or $\mathrm{KO}$ grew significantly slower than control tumors in SICD mice. These results are in line with recent findings proposing $L n c-T H O R$ as a novel therapeutic oncotarget for many human cancers ${ }^{16-20,45}$.

MAGE-TRIM28 complex is a cancer-specific AMPK $\alpha 1$ ubiquitin ligase en $^{21,27,40,41}$. We have previously shown that MAGEA6, one of the key AMPKal's ubiquitin ligase $^{21,27,40,41}$, is uniquely expressed in human glioma tissues and cells, responsible for AMPK $\alpha 1$ degradation and AMPK inhibition. Contrarily, MAGEA6 silencing/depletion restored AMPK $\alpha 1$ expression and induced AMPK activation, causing downstream mTORC1 inactivation and glioma cell death ${ }^{21}$. The regulatory mechanism of MAGEA6 expression in glioma is elusive. The results of this study suggest that LncTHOR-IGF2BP1 association is important for MAGEA6 expression in glioma cells. The RIP results show that MAGEA6 $m R N A$ directly binds to the IGF2BP1 protein in A172 cells and the primary glioma cells. Significantly, LncTHOR silencing/KO or IGF2BP1 KO induced MAGEA6 degradation (both mRNA and protein), AMPK $\alpha 1$ protein accumulation, and AMPK activation in A172 glioma cells. These results suggest that $L n c-T H O R-I G F 2 B P 1$ complex is important for MAGEA6 expression, causing AMPK 1 degradation and AMPK inactivation in human glioma cells.

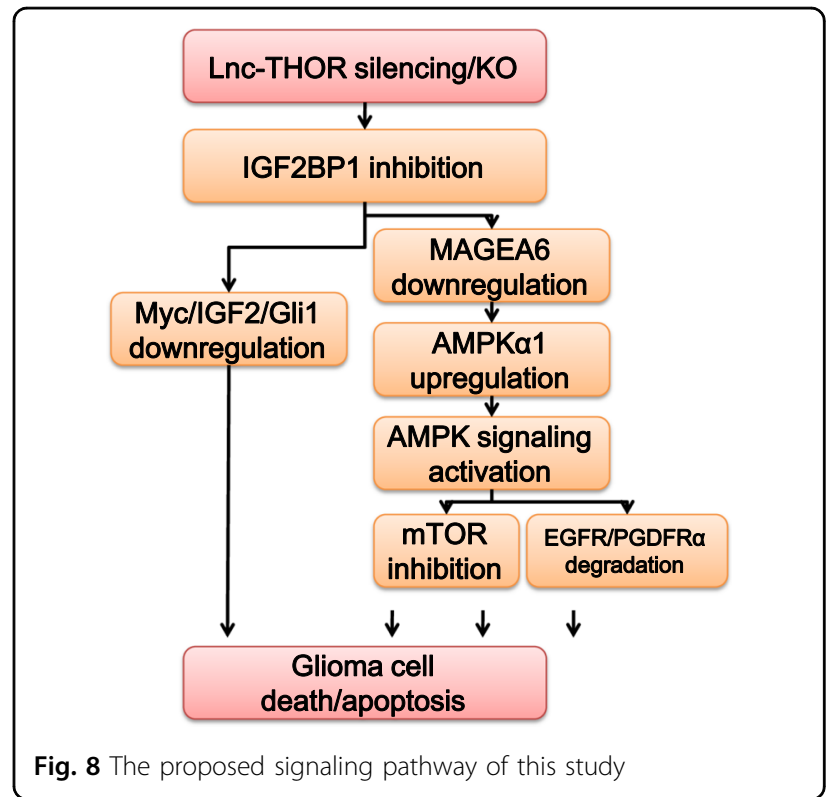

Our study ${ }^{21}$ and others have implied that forced activation of AMPK signaling can induce human cancer cell apoptosis via regulating its downstream effectors, including mTORC1 inhibition ${ }^{23,24,42}$, autophagy induction ${ }^{42-44}$, and RTK (EGFR, PDGFR, etc) degradation ${ }^{10,26}$. We provided evidences here to support that AMPK activation mediates, at least in part, Lnc-THOR-depletion-induced glioma cell death. In A172 cells, Lnc-THOR silencing/KO induced MAGEA6 degradation, AMPK $\alpha 1$ elevation, and AMPK signaling activation, causing mTORC1 inhibition and EGFR-PDGFR degradation and eventually cell apoptosis. Similarly, IGF2BP1 KO also activated MAGEA6-AMPK signaling in A172 cells. Importantly, AMPK inactivation, by AMPK $\alpha 1$ shRNA, KO, or dominant-negative mutation, attenuated Lnc-THOR shRNA-induced A172 cell apoptosis. Significantly, AMPK blockage failed to completely reverse Lnc-THOR shRNA-induced cytotoxicity in A172 glioma cells, suggesting that both AMPK-dependent and AMPKindependent mechanisms are responsible for Lnc-THORsilencing-induced glioma cell death (see Fig. 8, the proposed signaling pathway of the study). Therefore, although further studies are needed to explore the detailed underlying mechanisms, here we propose that Lnc-THOR-IGF2BP1 association is vital for MAGEA6 expression and AMPK inactivation in human glioma cells (Fig. 8).

\section{Conclusion}

Lnc-THOR depletion activates MAGEA6-AMPK signaling and inhibits human glioma cell survival.

\section{Acknowledgements}

This project was supported by the Fund of Shanghai Municipal Health Bureau (201640210), the Fund of Shanghai Charitable Cancer Research Center, and the Medical Cross Foundation of Shanghai Jiao-Tong University (YG2016MS59) all 
to S.-J.P. The funders had no role in study design, data collection and analysis, decision to publish, or preparation of the manuscript.

\section{Authors' contributions}

All listed authors designed the study, performed the experiments and the statistical analysis, and wrote the manuscript. All authors have read the manuscript and approved the final version.

\section{Conflict of interest}

The authors declare that they have no conflict of interest.

\section{Publisher's note}

Springer Nature remains neutral with regard to jurisdictional claims in published maps and institutional affiliations.

Received: 11 April 2019 Revised: 2 September 2019 Accepted: 9 September 2019

Published online: 14 November 2019

\section{References}

1. Siegel, R. L., Miller, K. D. \& Jemal, A. Cancer statistics, 2016. CA Cancer J. Clin. 66 7-30 (2016).

2. Siegel, R. L., Miller, K. D. \& Jemal, A. Cancer statistics, 2015. CA Cancer J. Clin. 65, 5-29 (2015).

3. Siegel, R., Ma, J., Zou, Z. \& Jemal, A. Cancer statistics, 2014. CA Cancer J. Clin. 64, 9-29 (2014).

4. Westphal, M. \& Lamszus, K. The neurobiology of gliomas: from cell biology to the development of therapeutic approaches. Nat. Rev. Neurosci. 12, 495-508 (2011).

5. Wen, P. Y. \& Reardon, D. A. Neuro-oncology in 2015: progress in glioma diagnosis, classification and treatment. Nat. Rev. Neurol. 12, 69-70 (2016)

6. Reardon, D. A. \& Wen, P. Y. Glioma in 2014: unravelling tumour heterogeneityimplications for therapy. Nat. Rev. Clin. Oncol. 12, 69-70 (2015).

7. Pan, S. J. et al. Tetraspanin 8-rictor-integrin alpha3 complex is required for glioma cell migration. Int. J. Mol. Sci. 16, 5363-5374 (2015).

8. Pan, S. J. et al. Ubiquitin-protein ligase E3C promotes glioma progression by mediating the ubiquitination and degrading of Annexin A7. Sci. Rep. 5, 11066 (2015).

9. Pan, S. J. et al. Over-expression of tetraspanin 8 in malignant glioma regulates tumor cell progression. Biochem. Biophys. Res. Commun. 458 476-482 (2015).

10. He, X. Y. et al. Gambogic acid induces EGFR degradation and Akt/mTORC1 inhibition through AMPK dependent-LRIG1 upregulation in cultured U87 glioma cells. Biochem. Biophys. Res. Commun. 435, 397-402 (2013).

11. Park, J. Y. et al. Roles of long non-coding RNAs on tumorigenesis and glioma development. Brain Tumor Res. Treat. 2, 1-6 (2014).

12. Li, Y. et al. Circular RNA as a biomarker for cancer: a systematic meta-analysis. Oncol. Lett. 16, 4078-4084 (2018).

13. Hou, L. D. \& Zhang, J. Circular RNAs: an emerging type of RNA in cancer. Int. J. Immunopathol. Pharm. 30, 1-6 (2017)

14. Zhao, Z. J. \& Shen, J. Circular RNA participates in the carcinogenesis and the malignant behavior of cancer. RNA Biol. 14, 514-521 (2017).

15. Hosono, Y. et al. Oncogenic role of THOR, a conserved cancer/testis long noncoding RNA. Cell 171, 1559.e20-1572.e20 (2017).

16. Ye, X. T., Huang, H., Huang, W. P. \& Hu, W. L. LncRNA THOR promotes human renal cell carcinoma cell growth. Biochem. Biophys. Res. Commun. 501 661-667 (2018).

17. Song, $H$. et al. LncRNA THOR increases the stemness of gastric cancer cells via enhancing SOX9 mRNA stability. Biomed. Pharmacother. 108 338-346 (2018).

18. Shang, Y. LncRNA THOR acts as a retinoblastoma promoter through enhancing the combination of c-myc mRNA and IGF2BP1 protein. Biomed. Pharmacother. 106, 1243-1249 (2018).

19. Cheng, Z. et al. Long non-coding RNA THOR promotes liver cancer stem cells expansion via beta-catenin pathway. Gene 684, 95-103 (2018).
20. Chen, W. et al. Long non-coding RNA THOR promotes human osteosarcoma cell growth in vitro and in vivo. Biochem. Biophys. Res. Commun. 499, 913-919 (2018).

21. Pan, S. J. et al. MAGEA6 promotes human glioma cell survival via targeting AMPKalpha1. Cancer Lett. 412, 21-29 (2018).

22. Jiang, H. et al. GSK621 targets glioma cells via activating AMP-activated protein kinase signalings. PLOS ONE 11, e0161017 (2016).

23. Inoki, K. Zhu, T. \& Guan, K. L. TSC2 mediates cellular energy response to control cell growth and survival. Cell 115, 577-590 (2003).

24. Inoki, K. et al. TSC2 integrates Wnt and energy signals via a coordinated phosphorylation by AMPK and GSK3 to regulate cell growth. Cell $\mathbf{1 2 6}$, 955-968 (2006).

25. Jones, R. G. et al. AMP-activated protein kinase induces a p53-dependent metabolic checkpoint. Mol. Cell 18, 283-293 (2005).

26. Chen, M. B. et al. Itraconazole-induced inhibition on human esophageal cancer cell growth requires AMPK activation. Mol. Cancer Ther. 17, 1229-1239 (2018).

27. Pineda, C. T. et al. Degradation of AMPK by a cancer-specific ubiquitin ligase. Cell 160, 715-728 (2015)

28. Cui, Y., Zhao, J., Yi, L. \& Jiang, Y. microRNA-153 targets mTORC2 component Rictor to inhibit glioma cells. PLOS ONE 11, e0156915 (2016).

29. Liu, Y. Y. et al. microRNA-200a downregulation in human glioma leads to Galphai1 over-expression, Akt activation, and cell proliferation. Oncogene $\mathbf{3 7}$ 2890-2902 (2018)

30. Cai, S. et al. Galphai3 nuclear translocation causes irradiation resistance in human glioma cells. Oncotarget 8, 35061-35068 (2017).

31. Li, Z. W. et al. Over-expression of Galphai3 in human glioma is required for AktmTOR activation and cell growth. Oncotarget https://doi.org/10.18632/ oncotarget.10995 (2016).

32. LV, Y. et al. Overexpression of lymphocyte antigen 6 complex, locus $E$ in gastric cancer promotes cancer cell growth and metastasis. Cell. Physiol. Biochem. 45 1219-1229 (2018).

33. Yang, L. et al. C6 ceramide dramatically enhances docetaxel-induced growth inhibition and apoptosis in cultured breast cancer cells: a mechanism study. Exp. Cell Res. 332, 47-59 (2015).

34. Li, K. R. et al. Ginsenoside Rg-1 protects retinal pigment epithelium (RPE) cells from cobalt chloride $(\mathrm{CoCl} 2)$ and hypoxia assaults. PLOS ONE 8 , e84171 (2013).

35. Lee, M. et al. AMP-activated protein kinase activity is critical for hypoxiainducible factor- 1 transcriptional activity and its target gene expression under hypoxic conditions in DU145 cells. J. Biol. Chem. 278 39653-39661 (2003).

36. Liu, Z. et al. IGF2BP1 over-expression in skin squamous cell carcinoma cells is essential for cell growth. Biochem. Biophys. Res. Commun. 501, 731-738 (2018).

37. Wu, F. et al. miR-1273g silences MAGEA3/6 to inhibit human colorectal cancer cell growth via activation of AMPK signaling. Cancer Lett. 435, 1-9 (2018).

38. Wang, Z. et al. IncRNA epigenetic landscape analysis identifies EPIC1 as an oncogenic IncRNA that interacts with MYC and promotes cell-cycle progression in cancer. Cancer Cell 33, 706-720 e709 (2018).

39. Wang, S. S. et al. Triptonide inhibits human nasopharyngeal carcinoma cell growth via disrupting Lnc-RNA THOR-IGF2BP1 signaling. Cancer Lett. $\mathbf{4 4 3}$ 13-24 (2019).

40. Pineda, C. T. \& Potts, P. R. Oncogenic MAGEA-TRIM28 ubiquitin ligase downregulates autophagy by ubiquitinating and degrading AMPK in cancer. Autophagy 11, 844-846 (2015).

41. Ye, X., Xie, J., Huang, H. \& Deng, Z. Knockdown of MAGEA6 activates AMPactivated protein kinase (AMPK) signaling to inhibit human renal cell carcinoma cells. Cell. Physiol. Biochem. 45, 1205-1218 (2018).

42. Kim, J., Kundu, M., Viollet, B. \& Guan, K. L. AMPK and mTOR regulate autophagy through direct phosphorylation of Ulk1. Nat. Cell Biol. 13, 132-141 (2011).

43. Egan, D. F. et al. Phosphorylation of ULK1 (hATG1) by AMP-activated protein kinase connects energy sensing to mitophagy. Science $\mathbf{3 3 1}$ 456-461 (2011)

44. Huo, H. Z. et al. AMP-activated protein kinase (AMPK)/Ulk1-dependent autophagic pathway contributes to C6 ceramide-induced cytotoxic effects in cultured colorectal cancer HT-29 cells. Mol. Cell. Biochem. 378, 171-181 (2013).

45. Ling, G. A. O., Xiu-Lian, C. \& Hua, C. A. O. LncRNA THOR attenuates cisplatin sensitivity of nasopharyngeal carcinoma cells via enhancing cells stemness. Biochimie 152, 63-72 (2018). 